\title{
Basic research of the consideration of additive manufactured lattice structures under thermo- and fluid dynamic loads
}

\author{
Karim Abbas ${ }^{1, *}$, Laura Thurn ${ }^{1}$, Julia Kessler $^{2}$ and Fabian Eichler ${ }^{1}$ \\ ${ }^{1}$ Aachen University of applied sciences, Faculty of Mechanical Engineering and Mechatronics, \\ Goethestr. 1, 52064 Aachen, GER \\ ${ }^{2}$ Institut für werkzeuglose Fertigung $\mathrm{GmbH} /$ Institute for toolless fabrication (IwF), Campus- \\ Boulevard 79, 52074 Aachen, GER
}

\begin{abstract}
This scientific research deals with lattice structures manufactured with laser powder bed fusion. Laser powder bed fusion is part of additive manufacturing. The so called layered construction is an increasingly used innovative manufacturing process that can be used to realize new design possibilities. Lightweight structures or bionic structures play a key role here. The focus of this work is on periodic lattice structures. In addition to saving resources and reducing the weight of components, lattice structures have particularly pronounced mechanical properties. However, little is known about their thermo- and fluid-dynamic properties. This work describes the first influencing factors of lattice structures in a thermo- and fluid-dynamic context using a case study. The aim of this paper is to evaluate important design and simulation criteria of lattice structures. Different lattice structures are considered, whose strut geometry is varied. The case study is carried out using a heat exchanger. While classical heat exchangers have lamellar structures, the substitution of these by lattice structures is evaluated. This work represents a first consideration of the most important parameters and gives an overview of the most important core points.
\end{abstract}

\section{Introduction}

This preliminary investigation is a primary work which investigates the thermo- and fluid dynamic behaviour of lattice structures.

Heat development and heat dissipation are an issue within machines and engines. In the following, the optimisation of a heat exchanger is discussed as an example. This is done with reference to the constant changes in the automotive industry, which requires lighter and more efficient components.

Additive manufacturing processes, also known under the general term $3 \mathrm{D}$ printing, which are based on the layer construction principle, are therefore increasingly finding their

\footnotetext{
* Corresponding author: abbas $@$,fh-aachen.de
} 
way into various industrial sectors. With this process it is possible to produce almost all geometries in one process. The focus of this research work is on periodic lattice structures, which represent a special form of lightweight structures. [1-5]

In addition to weight-reducing properties and their defined mechanical properties, lattice structures act as filters or bone frameworks in the field of medical technology. The research focus discussed in this scientific work deals with the evaluation of thermo- and fluid dynamic properties of lattice structures. The following heat exchange system is a fluid cooler which is defined by a cross flow of a liquid and a gaseous medium (water/air).

The modification of the cooling geometry is realized by the manufacturing possibilities of the additive manufacturing processes. Past and current research works also deal with the geometrical freedom and the possibilities of increasing the efficiency of heat exchangers in the field of layered construction processes. In the process, design changes are made, different structures are tested and different materials are evaluated. Saltzman et al. [5] compared conventional aircraft radiators with additive radiators. The basic dimensions of the innovative cooler have been adapted to the conventional cooler to ensure better comparability. In the course of the research work, the classic lamella structures were supplemented by additional openings in the lamellas and ventilation grilles. A comparison of the various coolers showed that the additive manufactured heat exchangers had up to $10 \%$ better heat conduction. At the same time, the pressure drop on the air side doubled. A similar study by Katheryn L. [6] et al. opened up improved heat conduction properties through the integration of wavy ducts. For this purpose different wavelengths of the channels were investigated. In addition to the comparison of straight and wavy channels, the influence of surface roughness on heat conduction was investigated. The investigation showed that the pressure loss increased with higher surface roughness, but the heat conduction also increased. While this research focused on the practical evaluation of the structural changes, the study by Brandon et al. [7] dealt with the integration of complicated structures as well as with the computer-aided simulation of these. An oil cooler was completely redesigned and AM-manufactured. Both the oil ducts, which among other things had lenticular cross-sections, and the finned tubes were adapted. Part of the work deals with CFD analysis, where the flow velocity, the pressure loss and the temperature curve were evaluated. This work also confirms the better heat conduction, which here was even up to $18 \%$ higher than that of a comparable classic cooler. However, higher pressure losses on the air side can also be observed here. Beyond the thermo- and fluid dynamic processes, the work deals with the constructional restrictions and possibilities to produce complete coolers AM-compatible. The researches which are described are based on aluminum alloys, which are suitable for use as heat exchangers due to their predestined heat conduction properties. Handler et al. [8, 9] pursue a different research approach, comparing both a stainless steel (316L) and an aluminum alloy (A16061). Part of the experimental setup is the evaluation of different pin geometries. These include elliptical, cylindrical as well as three-dimensional periodic lattice structures. As expected, the stainless steel showed lower performance in terms of thermal conductivity. However, the comparison of the different pin structures showed significant differences in relation to pressure loss and heat conduction. The lowest pressure loss was found with elliptical pins, while the cylindrical pins caused the highest pressure loss. The grid structure and the square webs had similar characteristics. However, despite the larger surface area, the heat conduction of the grid structures was lower. The following research work follows on from this aspect. Grid structures with different web geometries but the same unit cell are considered.

The most important requirements for the coolers are the largest possible surface area for heat dissipation, low air resistance and the generation of turbulence within the structure to improve the convection process and accelerate the removal of heat from the system. Grid structures, have large surfaces in a small installation space and, depending on the packing density of the cell, are flowable objects. This is due to the open cell structure, which ensures 
permeability. Due to the branched structures of the cell, flow-changing measures can be initiated which cause turbulence. Since there is almost no scientific knowledge on the turbulence generation of lattice structures, this scientific work deals with the basics of the flow theory of lattice structures [10-16].

\section{Methods}

The experimental setup is divided into three main sections: simulation, design and prototype testing. At the beginning of the experiment, the flow behaviour of three different lattice structures are simulated using CFD analysis (Computational Fluid Dynamics - CFD). The foundation of the test series is formed by different elementary cells of a lattice structure. These only differ in the geometry of the struts. A circular, square and triangular cross-section is considered. Research experiments by Kessler [17], who in her research experiments investigated different web cross-sections for different mechanical loads, e.g. tensile, compressive and continuous vibration loads, form the basis for this. In order to be able to compare the webs, they have the same cross-sectional area of $1 \mathrm{~mm}^{2}$. The cell size is fixed at $5 \times 5 \times 5 \mathrm{~mm}$. Since there are no empirical values regarding the cell size for the flow behaviour of grid structures, the cell size is freely chosen. However, this is based on previously prepared test series and scientific work. The main objective of the digital simulation is the thermo fluid dynamic simulation of a cooler, which is flowed through by a tempered hot water flow and is surrounded by an air flow and cooled. A ride with an automobile is simulated. For experimental purposes, an inner-city average speed of the vehicle is selected. In order to carry out the actual simulation of the optimised end product, preliminary tests must be carried out in advance. With the help of the CFD simulation previously carried out, it is finaly possible to design a cooler. The main aim is to determine the cooling capacity of two 3D-printed coolers made out of $316 \mathrm{~L}$ stainless steel. First and foremost, it is a matter of measuring a temperature difference. For a better understanding, the cooler is regarded as a system surrounded by a system boundary. A mass flow with an input temperature $\mathrm{T} 1$ is fed into the cooler system. At the same time a mass flow with a temperature T2 is led out again. Parallel to the water flow, an air flow is fed into the system (T3-In; T4-Out). The measurements of these two temperature differences provide the basis for the series of experiments. A turbomachine produces an airflow to simulate a driving speed of $50 \mathrm{~km} / \mathrm{h}$.

\section{Thermo and fluid dynamics}

The core element of the scientific work is the simulation of lattice structures and the gaining of knowledge about fluidic behaviour using "Autodesk CFD 2019 program". For this purpose, the main elements of lattice structures and the elementary cells are categorically investigated and tested. Starting with the lattice bars and their effects on the flow behaviour of fluids followed by testing the unit cells.

In order to link up with the requirement for heat transport, it should be kept in mind that a good flow around and rapid removal of the heated air is preferred. Furthermore, slight turbulences should favour convection. The fist step is to flow around individual rods in a test tunnel. Followed by a simulation of unit cells. These simulations are the basis for the first simulations of cell walls. In the course of the investigation, the number of cell walls will be determined. Pressure losses and flow velocities are considered. From this, conclusions are drawn as to which web geometry and cell structure are best suited for the design of a cooler. The simulation of single cell walls show slight differences in presser drops and flow velocities.

The next juxtaposition is the simulation of a second cell row and on the right in the image directly a three-row cell structure (Fig. 1). It becomes clear that there is almost no 
change in the flow pattern, regardless of the shape of the web. Thus, it is not necessary to increase the number of series and to carry out further simulations. In all three pictures the velocity fields with corresponding velocity vectors are shown. The vectors are indicators for the direction of the flow. It can be seen that with all three cell types there are strong turbulences in the areas of the elongated blue fields. In the case of the circular structure and the triangular structure, however, it is obvious that there are agglomerations of velocity levels, while the velocity profile of the quadrilateral webs is more homogeneous. Here the assumption is obvious that the passage cross-section of the nozzle profile does not change due to the straight web walls, while the flow at the circular cross-sections is guided in such a way that the vector directions represent a larger curve. The flow in the quadrilateral structure, on the other hand, is almost straight. In addition, it can be seen that the velocity of the flow is also higher in front of the grid structure. A higher velocity results in a lower dynamic pressure in front of the grid structure.

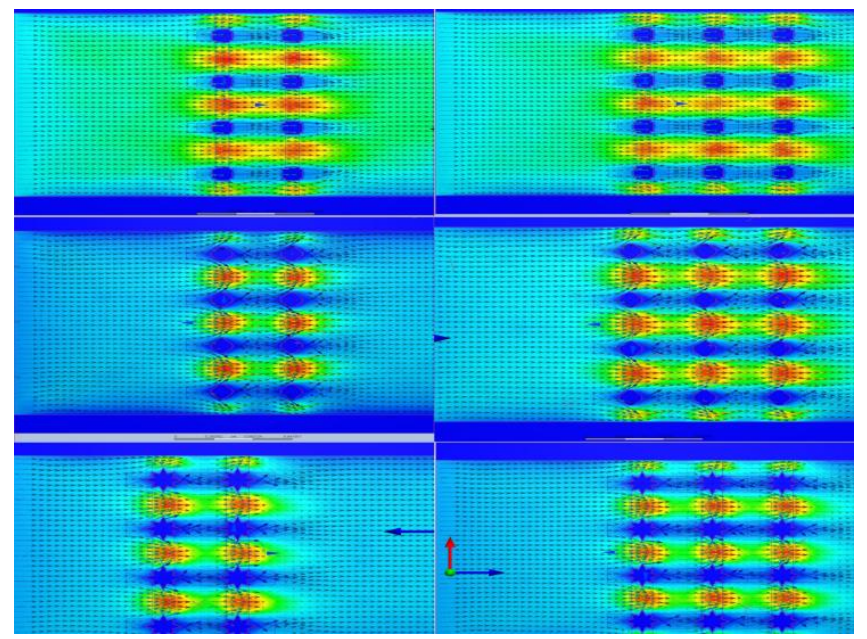

Fig. 1 Two and three row lattice structure (1.Square shape, 2. Circular shape, 3. Triangular shape).

These final preliminary analyses thus show that the flow pattern in a cell composite of elementary cells with a square cross-section is favoured by flow technology.

More homogeneous velocity profiles across the lattice structure can be observed. Since the heat transfer coefficient discussed above also increases with increasing flow velocity, the heat transport is positively influenced. Too high velocities, as they are present in the agglomeration of the other cell structures, cause too high pressure loss, which in turn has a negative effect on the heat transfer coefficient. With this grid structure, a homogeneous velocity profile as well as additional turbulences is now generated. These factors are part of the hypothesis for heat transfer optimization using lattice structures. However, this is not the only decision criterion for the selection. In addition to the flow properties, geometrical occurrences are also important parameters to be included. The consideration of volumes and surfaces should not be neglected.

Since the aim is to generate as large area as possible for the heat transfer and at the same time to keep the mass of the component low, these criteria should be taken into account for the selection. Here the triangular structure crystallizes as optimal, because with the largest surface and at the same time the smallest volume, the geometry is apparently optimal. When adding the simulation results, however, deficits in the flow behavior become apparent. The quadratic webs, on the other hand, show preferred flow properties and lie in 
the mid zone for the geometric properties. Consequently, a grid structure with a square cross-section is chosen for the design of the cooler.

The cooler is designed with the selected grid structure. For a simplified simulation a cooler segment is considered which consists of a water-bearing segment and the corresponding heat sink. A value of $50^{\circ} \mathrm{c}$ is selected for the input temperature, which will later also be used for real testing of the cooler. The flow velocity is set to $0.2 \mathrm{~m} / \mathrm{s}$ and for the air flow a velocity of $50 \mathrm{~km} / \mathrm{h}$ is set. The heat transfer is limited here to convection. The input temperature of the air is set to $30^{\circ} \mathrm{c}$. This temperature is also generated by the blower of the test apparatus, which is used for real testing.

Since the prototypes are printed from stainless steel, the same material parameters are also defined for the simulation. Thus all important test parameters are considered and integrated. Figure 2 shows the heat distribution within the cooler. As before, the integration of further cells does not change the flow pattern. The temperature distribution shows that the temperature of the water decreases over the entire cooling section. Even if only minimally, the temperature drops by three degree Celsius. This result is due to the poor heat conduction properties of stainless steel.

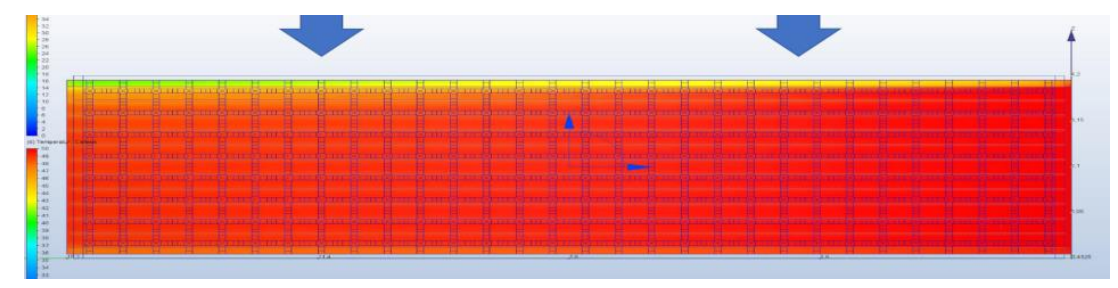

Fig. 2 CFD-analysis of heat exchanger module (velocity distribution/ heat distribution).

With the help of the knowledge gained from the simulation, it is now possible to design the coolers. Since a comparison of the prototype with a conventionally manufactured cooler is hardly possible due to the size, geometry and material used, an additional cooler is constructed. This cooler is based on a conventional cooler and also has fins in the air area as cooling geometry. In order to create an approximate comparability, the fins have a distance of $5 \mathrm{~mm}$. This is based on the size of the elementary cells, which also have an edge length of $5 \mathrm{~mm}$. The lamella thickness is limited to $1 \mathrm{~mm}$, as are the webs of the grid structures. Both coolers are generated with the SLM M1 Cusing from ConceptLaser (Fig. 3). The layer thickness is set to $45 \mu \mathrm{m}$ and the laser power is $150 \mathrm{~W}$. The protective gas used here is argon. The components are not reworked at all. Both components were then tested on a test bench.

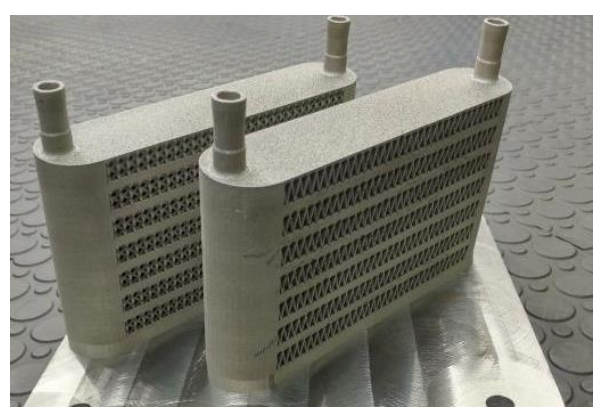

Fig. 3. AM heat exchangers: fine tubes (front), lattice structure (back). 
As previously explained in the test setup, temperature measurements were carried out at various points. The measurements are divided into three measurement series with the conventional cooler (M1-M3) and two measurement series with the grid structure cooler (M4-M5). Since the task is thermofluiddynamic processes in which a heat exchange takes place, the heat flows of the two fluids are shown in the first step of the analysis and evaluation. To assess the efficiency of the coolers to be compared (conventional and lattice structure coolers), the measure of the heat transfer coefficient $[\alpha]$ is indispensable. With this value it is possible to describe the quality of the energy transfer. The heat transfer coefficient can be calculated theoretically if the flow field is known. The determination or qualitative comparison of this parameter is the main objective of the analysis.

$$
\alpha=\frac{N u \cdot \lambda}{d_{i}}
$$

Depending on the flow behaviour (laminar or turbulent) the number of nuts $[\mathrm{Nu}]$ can be determined.

This applies to turbulent flow:

$$
N u_{d, t u r b}=\frac{\xi}{8} \cdot \frac{R e_{d_{i}} \cdot \operatorname{Pr}}{1+12,5 \cdot \sqrt{\frac{\xi}{8}}\left(\operatorname{Pr}^{2} \overline{3}-1\right)}\left(1+\left(\frac{d_{I}}{l}\right)^{\frac{2}{3}}\right)
$$

The Nusselt number $\mathrm{Nu}$ is the ratio of the occurring convective heat transfer (turbulent flow) to the pure heat conduction (laminar flow). It thus describes the ratio of the heat conduction of a turbulent flow in comparison with the same boundary conditions in which the flow is laminar. Since the heat conduction of a turbulent flow is always greater than that of a laminar flow, the number of Nusselt is always greater than or equal to $\mathrm{Nu}=1$.

The heat transfer coefficient of the water plays only a minor role in the investigations made here, since the water flow is exactly the same in both subsystems. In addition, the analysis of the water flow shows that the Reynolds number of the water flow is approx. Re $=126.13$.

The Reynolds number of the water flow is approx. $\mathrm{Re}=126.13$. This is an indication that the water flow is laminar. Since the heat transfer coefficient is dependent on the number of Nusselt last and the number of Nusselt in a laminar flow only depends on geometric and material-specific parameters, it can be assumed that the transition coefficient of the water is identical for both coolers. Therefore, the heat transfer coefficient of the water is not of interest for the evaluation of the coolers.

The core elements of the cooling systems are the lamella structure and the grid structure, which is surrounded by air. In these areas, the heat is transferred to the air and thus dissipated. Fig. 4 shows that the heat transfer coefficient of the air in the innovative grid structure cooler (measurement series M4 and M5) is significantly higher. Taking into account the equations already introduced, this leads to the assumption that the turbulence degree of the innovative cooler is significantly higher than that of the conventional finned cooler.

This, in turn, has a direct influence on the Nusselt number. The turbulence is generated by the grid structures. The assumption that the grid structures improve the cooling performance by increasing the turbulence is confirmed by this series of tests. Since this is the first series of experiments in this field, further basic research for verification and concretization is indispensable. 


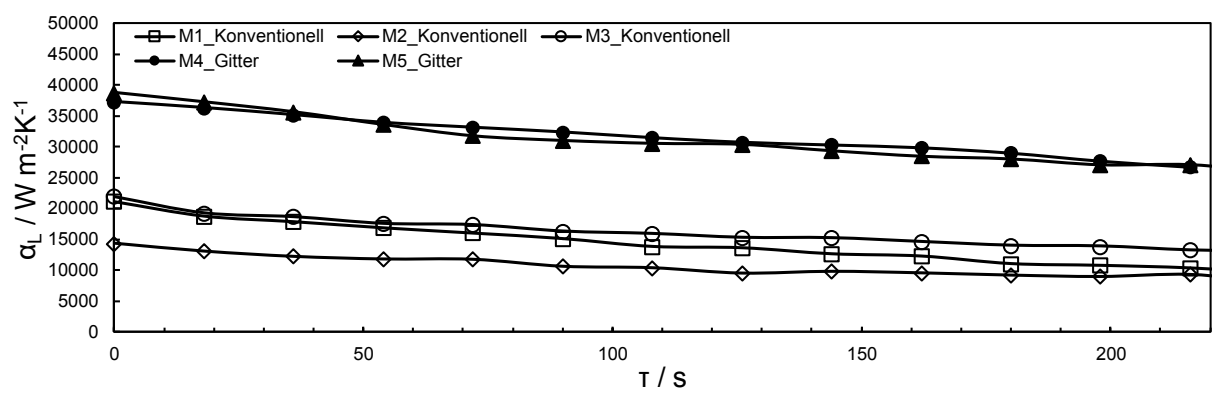

Fig. 4. Heat transfer coefficient (M1-M5).

\section{CONCLUSIONS}

The scientific work discussed here merely lays the foundation for further series of experiments and empirical research. In this work, lightweight construction was linked to the automotive industry, but has the potential to be adapted to other fields of application. The application was explained in simplified form using an engine cooler. Here, grid structures crystallized out as the preferred lightweight design strategy. Periodic lattice structures have hardly been researched and integrated into a cooler. First digital CFD simulations opened the potential of the cooling capacity. For a better comparison, a second cooler with a classic design was printed and was also part of the digital and real test series. The hypothesis to be investigated was more that with a large surface area of the heat sink and optimal flow, the cooling performance could be improved. It was shown in the previous CFD analyses as well as in the real tests that the grid structures have a large potential. Despite the small surface area for cooling, the heat transfer of the grid structures was larger than that of the lamella structure. This is due to the turbulent flow inside the cooler caused by the lattice structures themselves. This series of experiments thus provided first insights into the thermodynamic fluid behaviour of lattice structures. However, as this is a first pilot project, further optimizations have to be carried out during the execution of the experiments and in the construction in order to obtain well-founded and meaningful results. Simplified, the test series presents itself in three phases. The first phase was the CFD simulation, the basis for the design and manufacturing phase. The results were prototypes which were tested during the test phase.

The simulation was a decisive and fundamental process step. The first insights into the flow behaviour of lightweight structures could be gathered here. However, even more indepth tests have to be carried out, which test further types of lattice structures and continue to focus on deeper basic research. Thus, in the first simulations, vertical webs were flowed around. In the future, however, it would be appropriate to supplement these studies with simulations of angled webs in order to obtain more information about the later lattice structures. In addition, the CFD libraries are only equipped with characteristics of conventionally manufactured raw materials. Additives, however, exhibit fundamental differences in anisotropy and, for example, surface quality. Quality is a factor that should not be neglected. As a result, even more experience can be gained in this field.

The design and manufacture are also the main focuses of the test series that can be optimized. Starting with the selected material, it has become clear (although it was known before) that stainless steel is not an optimal material for a cooler. For the production of the aluminium radiator, the experience values regarding constructive measures were too low at that time. Therefore, stainless steel was chosen for simplified purposes. 


\section{References}

1. W. Hennig, A. Freialdenhoven, I. Olschewski I, Aufl., Wiesbaden, Vieweg Teubner Springer, (2009)

2. H. Legler, B. Gekrke, O. Krawczyk, Bundesministerium für Wirtschaft und Technologie (2009)

3. R. Golloch, Ein wirkungsvolles Konzept zur Kraftstoffverbrauchssenkung Springer, (2005)

4. M. Seeberg, Dissertation TU Bamberg, (2016)

5. D. Saltzmann, M. Bichnevicius, S. Lynch, T.W. Simpson, 55th AIAA Aerospace Sciences Meeting (2017)

6. L. K. Kirsch, et al., Journal of Turbomachinery, 139, (2017)

7. J. Hathaway, K. Garde, C. Susan, C. Mantell, J. Davidson, International Journal of Heat and Mass Transfer, 117, (2017)

8. E. Handler, A. Sterlin, J. Pegues, H. Ozdes, M. Masoomi, N. Shamsaei, M. Scott, Solid Freeform Fabrication Symposium, (2017)

9. D. Jafari, W.Wessel, W. Wits, Renewable and Sustainable Energy Reviews, 91, (2018)

10. E. Dondugan, Dissertation TU Bochum, (2001)

11. H. Herwig, A. Moschallski, Wärmeübertragung, Springer (2014)

12. O. Rehme, Dissertation TU Hamburg (2010)

13. H. Hasib, A. Rennie, N.Burns, L. Geekie, Lancester, (2015)

14. S. Teufelhart, Dissertation TU München, (2016)

15. A. Gebhardt, Generative Fertigungsverfahren, Aufl., München, Hanser, (2007)

16. N. Balc, S.C. Cosma, J. Kessler, V. Mager, Applied Mechanics and Materials, 808, 199-204, (2015)

17. J. Kessler, Research on improved lattice structure parts made by selective Laser Melting, PhD thesis TU Cluj-Napoca, (2017) 